Supporting Information

\title{
Effect of Post-deposition Annealing on the
}

\section{Luminescence of Mixed-phase $\mathrm{CsPb}_{2} \mathrm{Br}_{5} / \mathrm{CsPbBr}_{3}$}

\section{Thin Films}

\author{
Sebastián Caicedo-Dávila ${ }^{1}$, René Gunder ${ }^{1}$, José A. Márquez ${ }^{1}$, Sergiu Levcenko ${ }^{1}$, Klaus \\ Schwarzburg' ${ }^{2}$,Thomas Unold ${ }^{1}$, and Daniel Abou-Ras ${ }^{1}$ \\ ${ }^{1}$ Department Structure and Dynamics of Energy Materials, Helmholtz Zentrum Berlin for \\ Materials and Energy, Hahn-Meitner Platz 1, 10104, Berlin, Germany \\ ${ }^{2}$ Institute Solar Fuels, Helmholtz Zentrum Berlin for Materials and Energy, Hahn-Meitner Platz \\ 1, 10104, Berlin, Germany
}

Sample characterization: The phases in the coevaporated film were first identified by X-ray diffraction (Figure S1a). As discussed elsewhere, ${ }^{1}$ the film exhibits a strong preferred orientation of the $\mathrm{CsPbBr}_{3}$ phase. The PL spectrum (Figure $\mathrm{S} 1 b$ ) confirms the presence of the $\mathrm{CsPbBr} 3$ phase with a band-to-band transition at $2.35 \mathrm{eV}$, and the PL maps (Figure S1c) show and uneven distribution of the energy position of the transition and the luminescence intensity. The crosssectional correlative SEM analysis (Figure S1d) reveals that the phases are distributed in a layered 
fashion. Near the substrate, the PL active (in visible range) $\mathrm{CsPbBr}_{3}$, and towards the surface the $\mathrm{PL}$ inactive, $\mathrm{Cs}$ depleted, $\mathrm{CsPb}_{2} \mathrm{Br}_{5}$ phase.
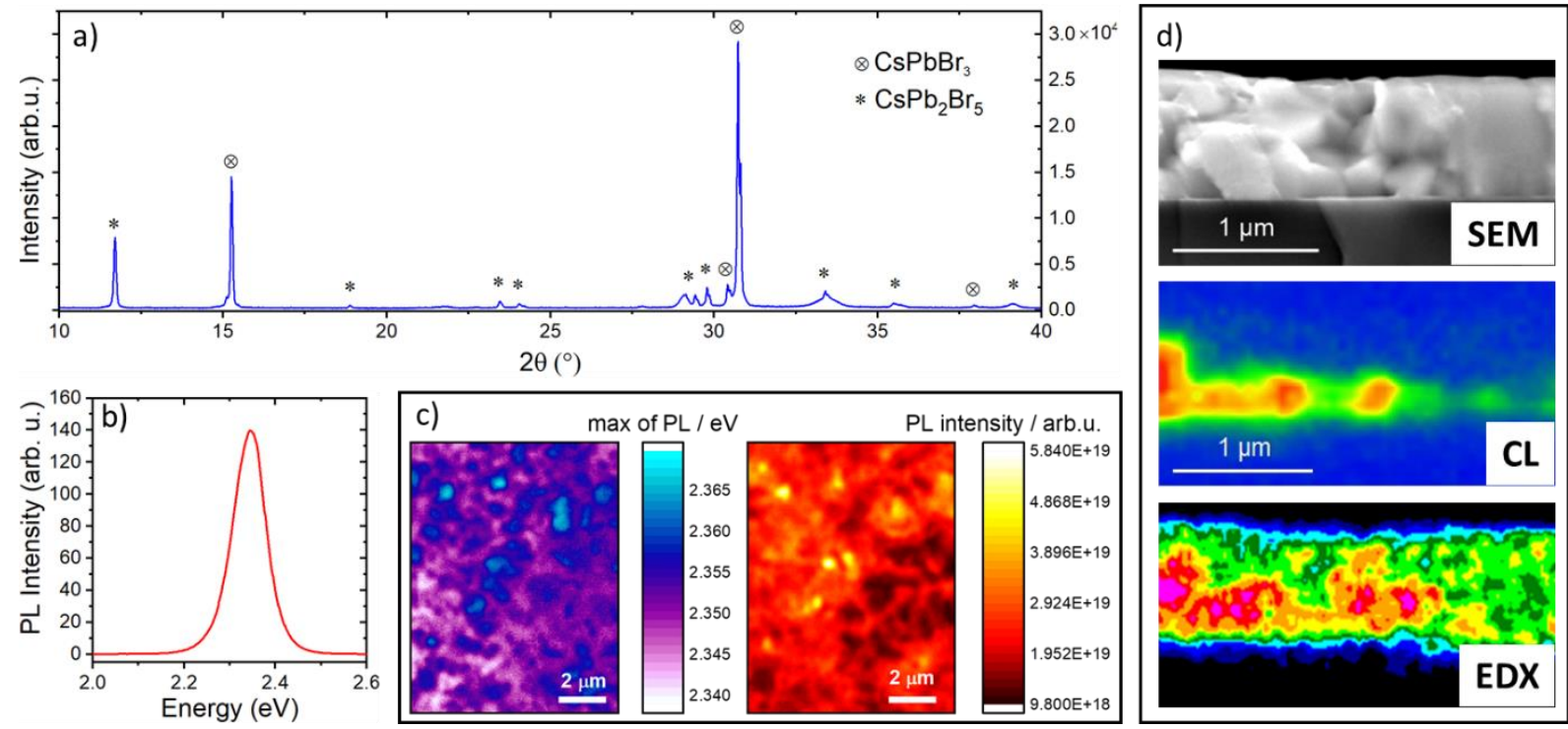

Figure $\mathrm{S} 1$. (a) XRD pattern of the coevaporated mixed-phase $\mathrm{Cs} \mathrm{PbBr}_{3} / \mathrm{Cs}_{2} \mathrm{~Pb}_{2} \mathrm{Br}_{5}$ film. The patterns were indexed using PDF 01-072-7929 $9^{2}$ for $\mathrm{CsPbBr}_{3}$ and $\mathrm{PDF} 00-025-0211^{3}$ for $\mathrm{CsPb}_{2} \mathrm{Br}_{5}$. (b) steady-state PL spectrum, (c) PL peak position and intensity maps of the film. (d) Microscopic characterization of the film's cross-section, including SEM image, CL and EDX on the same position.

\section{Experimental Details}

EDX composition maps were acquired at an acceleration voltage of $10 \mathrm{kV}$ and at a beam current of below $100 \mathrm{pA}$ to avoid beam damage to the film surface and cross-section surface. We identified the composition using Cs-L (4.287 and $4.620 \mathrm{keV}), \mathrm{Pb}-\mathrm{M}$ (2.345 and $2.443 \mathrm{keV})$, and Br-L (1.340 and $1.480 \mathrm{keV}) \mathrm{X}$-ray characteristic line series.

CL maps were acquired using an aluminum parabolic mirror positioned above the sample to focus the light onto a monochromator grating of 300 lines/mm blazed at $500 \mathrm{~nm}$, outside the SEM chamber. The CL maps were acquired at an acceleration voltage of $10 \mathrm{kV}$ and at a beam current of 50 pA. The dwelling time per pixel was adjusted between 20 and $100 \mathrm{~ms}$, ensuring good signal-tonoise ratio and avoiding detector saturation. The pixel size was set to $50 \mathrm{~nm}$ and a Zyla $5.5 \mathrm{sCMOS}$ 
camera from Andor was used to measure the CL spectra. We removed the background (beamblanked acquisition) and compensated for the system response in all the CL spectra and hyperspectral images were.

In-situ XRD measurements were carried out in a Bruker D8 powder diffractometer in a BraggBrentano configuration, using a $\mathrm{Cu} \mathrm{K} \alpha$ radiation source $(\lambda=0.15406 \mathrm{~nm}) .2 \theta$ was measured between 10 and 60 degrees with a step size of 0.016 degrees and a time per step of $96 \mathrm{~s}$. The sample was heated using a high temperature oven chamber in $\mathrm{N}_{2}$ atmosphere (1130 mbar) and thermal expansion of the measuring stage was compensated. 

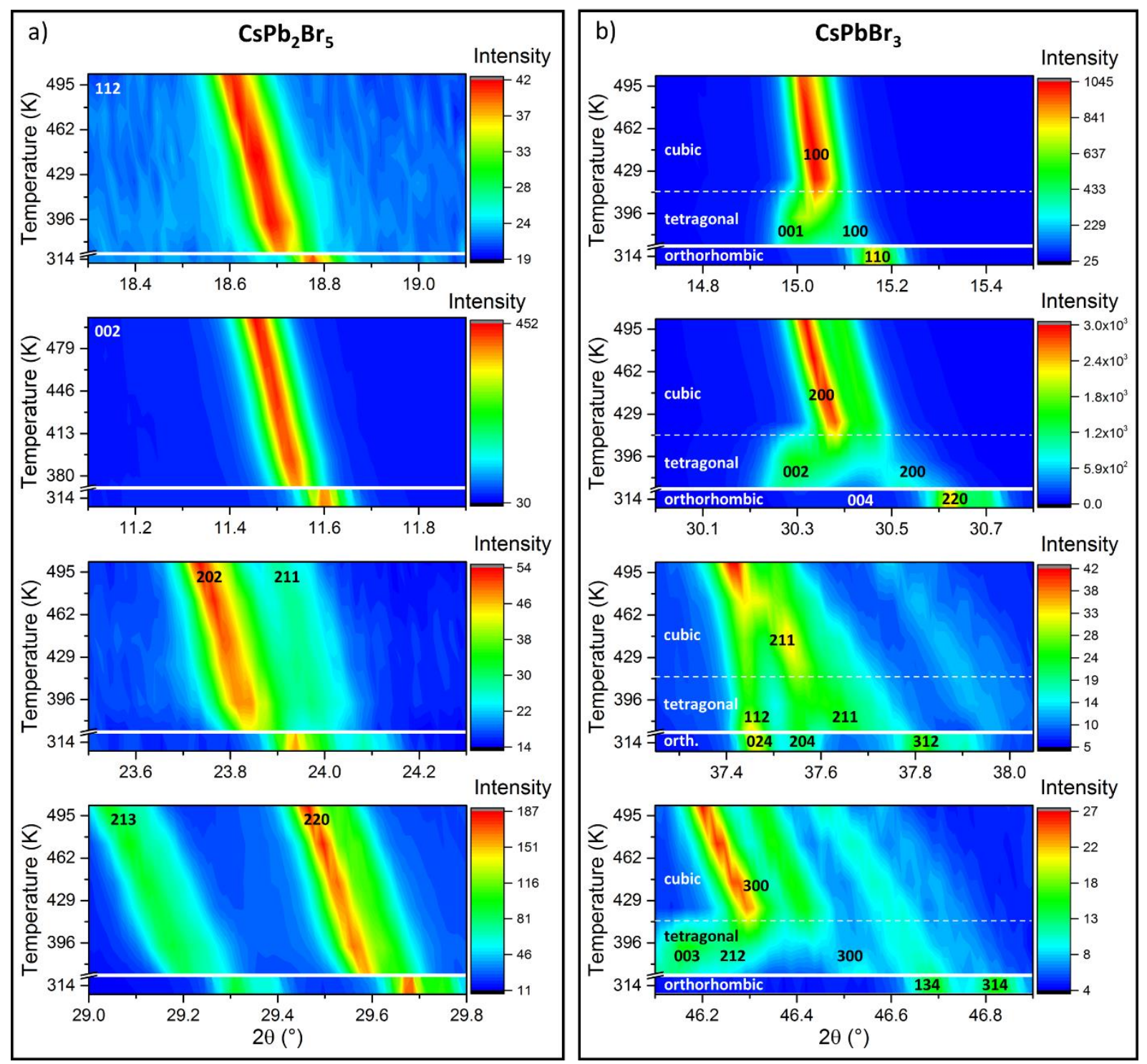

Figure S2. Contour maps showing the evolution with temperature of various diffraction peaks of (a) $\mathrm{CsPb}_{2} \mathrm{Br}_{5}$ and (b) $\mathrm{CsPbBr}_{3}$. The peaks are identified in the figure as well as the domains of orthorhombic, ${ }^{2}$ tetragonal, ${ }^{4}$ and cubic $^{2}$ phases of $\mathrm{CsPbBr} 3$. 

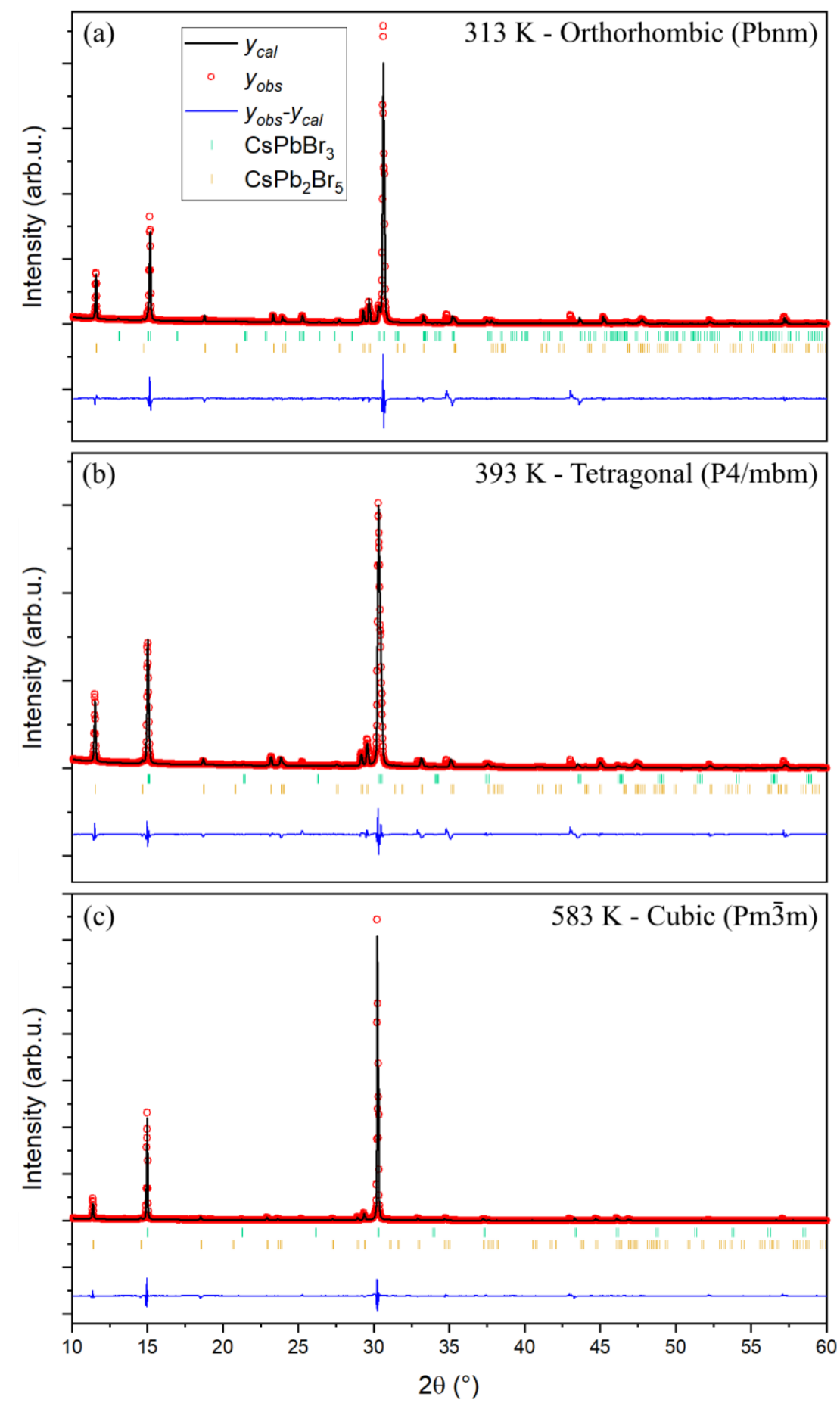

Figure S3. Illustrative results of LeBail refinement at three different temperatures for each of the structural phases of $\mathrm{CsPbBr}$ : (a) orthorhombic, (b) tetragonal and (c) cubic. 


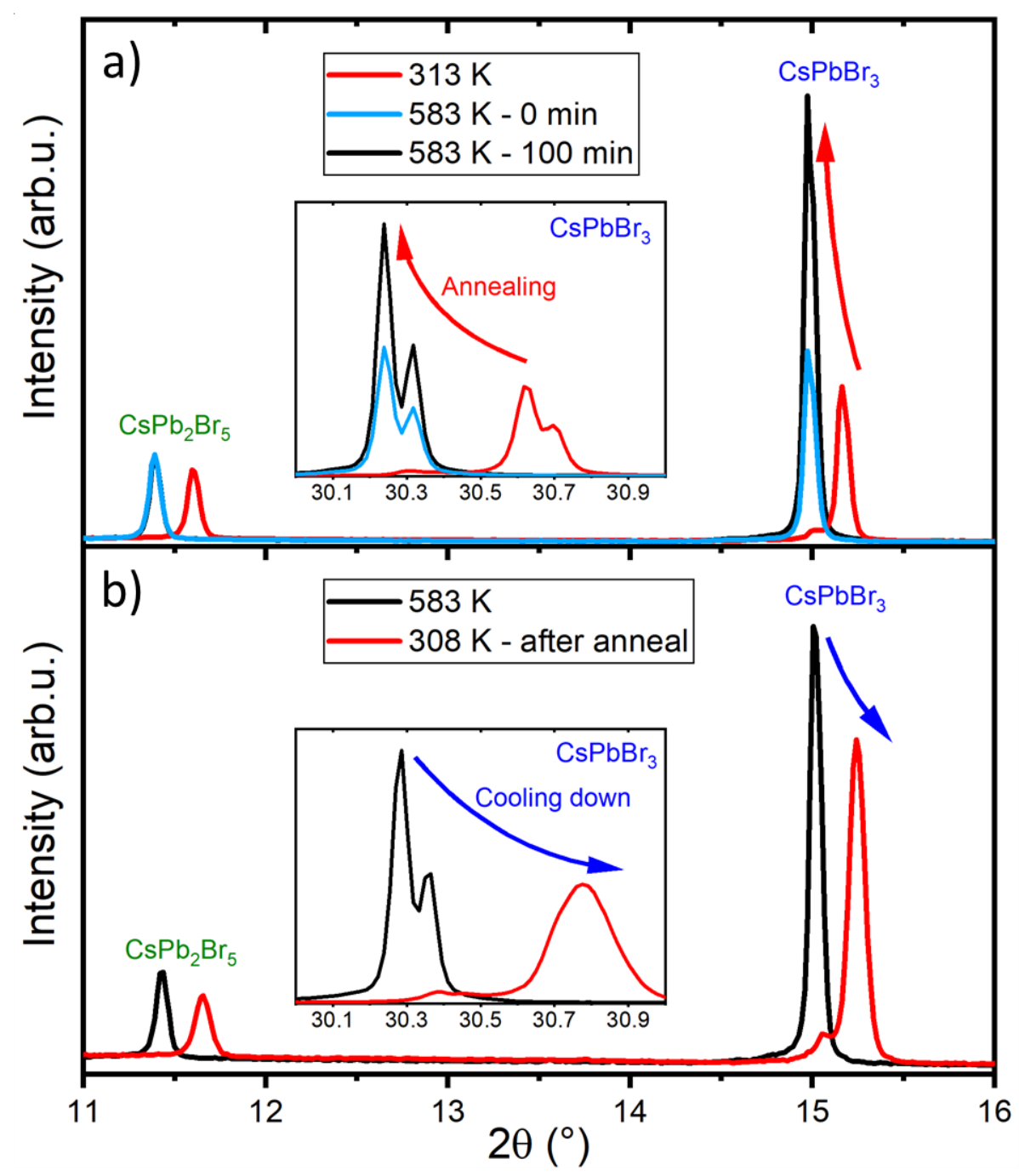

Figure S4. Changes in the intensity of the main $\mathrm{Bragg}$ reflection of $\mathrm{Cs}_{2} \mathrm{~Pb}_{2} \mathrm{Br}_{5}$ and $\mathrm{CsPbBr}_{3}$ (inset) measured during (a) $583 \mathrm{~K}$ annealing and (b) at $308 \mathrm{~K}$ after annealing. Note that after annealing, the intensity for the 002 reflection is reduced, while the ones for $\mathrm{CsPbr}_{3}$ effectively increases indicating a larger volume fraction. 


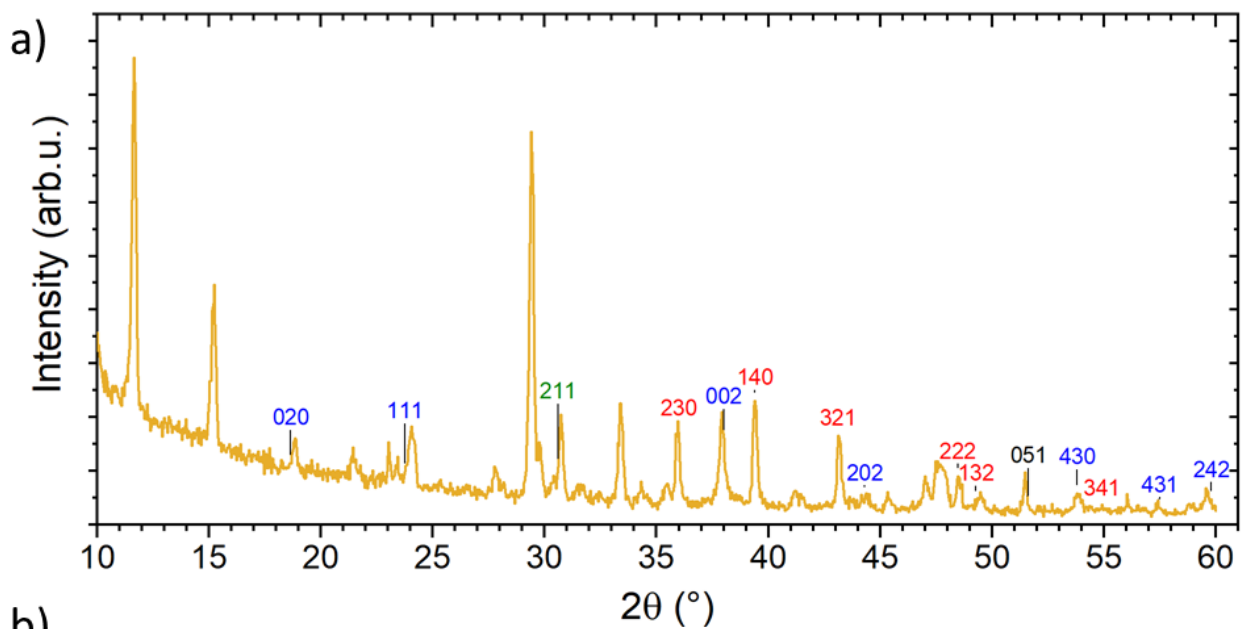

b)
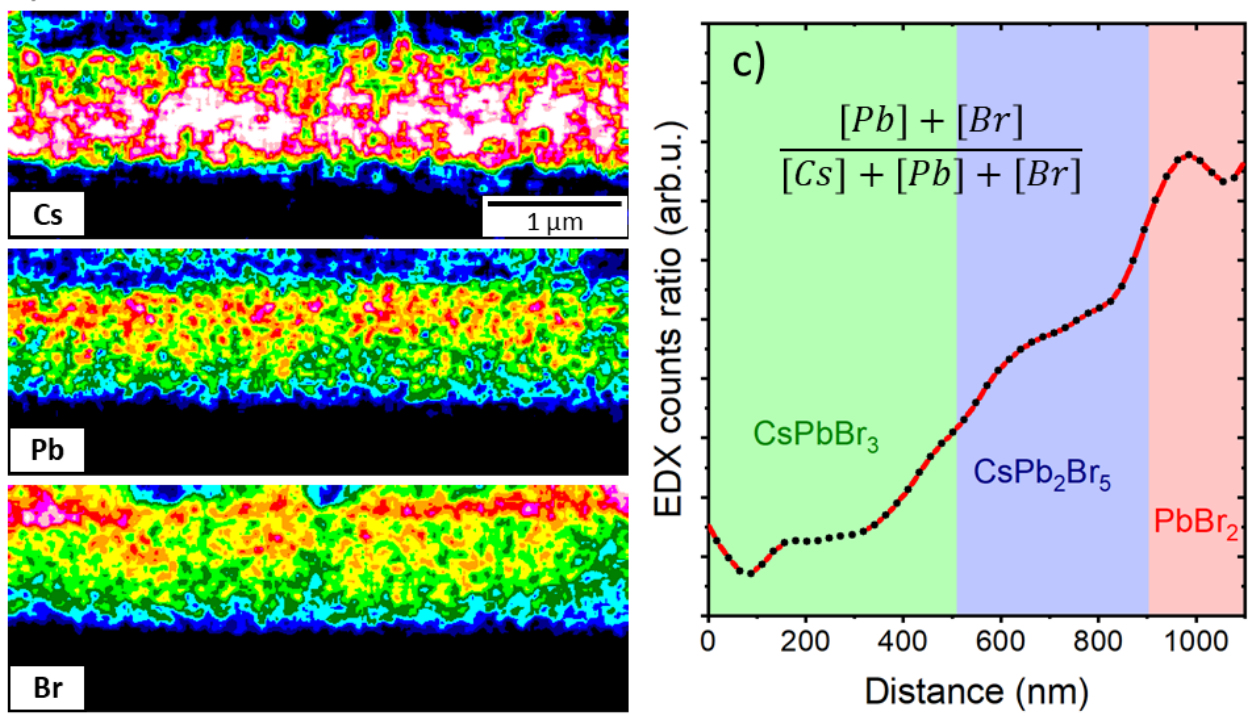

Figure S5. (a) GIXRD pattern of the mixed film, acquired after annealing. The reflections identified as $\mathrm{PbBr}_{2}$ are shown in the plot. Reflections in blue coincide with reflections of $\mathrm{CsPb}_{2} \mathrm{Br}$, and that in green coincides with a $\mathrm{CsPbBr}_{3}$ Bragg peak. The reflections in red are new reflections not observed in Bragg-Brentano configuration. (b) Cross-sectional EDX maps of $\mathrm{Cs}, \mathrm{Pb}$ and $\mathrm{Br}$ showing the enhanced signal of $\mathrm{Pb}$ and $\mathrm{Br}$ towards the surface. $(\mathrm{c})([\mathrm{Pb}]+[\mathrm{Br}]) /([\mathrm{Cs}]+[\mathrm{Pb}]+[\mathrm{Br}])$ EDX counts ratio, showing the increased concentration of $\mathrm{PbBr}_{2}$ towards the surface of the film. The position of the different phases are shown in colored areas. 


\section{Discussion of the surface CL measurements}

To interpret the results of the surface CL maps and accumulated spectra correctly, we have to take into account how the electron beam excites the surface of the sample. The penetration depth of the electron beam is limited, and we can estimate it as a simple function of the density, ${ }^{5}$ in which $R_{e}=0.398 E^{1.75} / \rho$, where $R_{e}$ is the penetration depth in $\mathrm{nm}, E$ is the electron beam energy in $\mathrm{keV}$, and $\rho$ the mass density of the sample in $\mathrm{g} / \mathrm{cm}^{3}$. Using the density of the top layer $\mathrm{CsPb}_{2} \mathrm{Br}_{5},{ }^{6}$ $\rho=11.48 \mathrm{~g} / \mathrm{cm}^{3}$, we estimate a $R_{e} \sim 195 \mathrm{~nm}$ from the surface of the film for an acceleration voltage of $10 \mathrm{kV}$; this means that, on grain interiors, the electron beam only excites the $\mathrm{CsPb}_{2} \mathrm{Br}_{5}$ on top. Figure $5 \mathrm{c}$ in the main text shows that the CL exhibits its maximum intensity in the regions of green emission $(2.35 \mathrm{eV}$, Figure $5 \mathrm{a}$ in the main text). These are regions between grains, where the electron beam penetrates deeper into the film, exciting the $\mathrm{CsPbBr}_{3}$ bottom layer.

Figure S6 shows a schematic band diagram of the radiative recombination mechanisms in PL and CL experiments. As discussed in the main text, the laser in PL experiments cannot excite the surface $\mathrm{CsPb}_{2} \mathrm{Br}_{5}$ layer, owing to its large bandgap $(>3.0 \mathrm{eV})$. This means that the measured $\mathrm{PL}$ signal originates from the $\mathrm{CsPb}_{2} \mathrm{Br}_{5} / \mathrm{CsPbBr}_{3}$ interface, where it is absorbed by the $\mathrm{CsPbBr}$. In the contrary, the high-energy electrons in CL, can (among many other interactions) generate electronhole pairs in the $\mathrm{CsPb}_{2} \mathrm{Br}_{5}$. These can recombine radiatively via band-to-band recombination which we are unable to detect - and via deep defect levels, which originate the measured $1.8 \mathrm{eV}$ band, according to the defect positions predicted by Yin et al. ${ }^{7}$ 

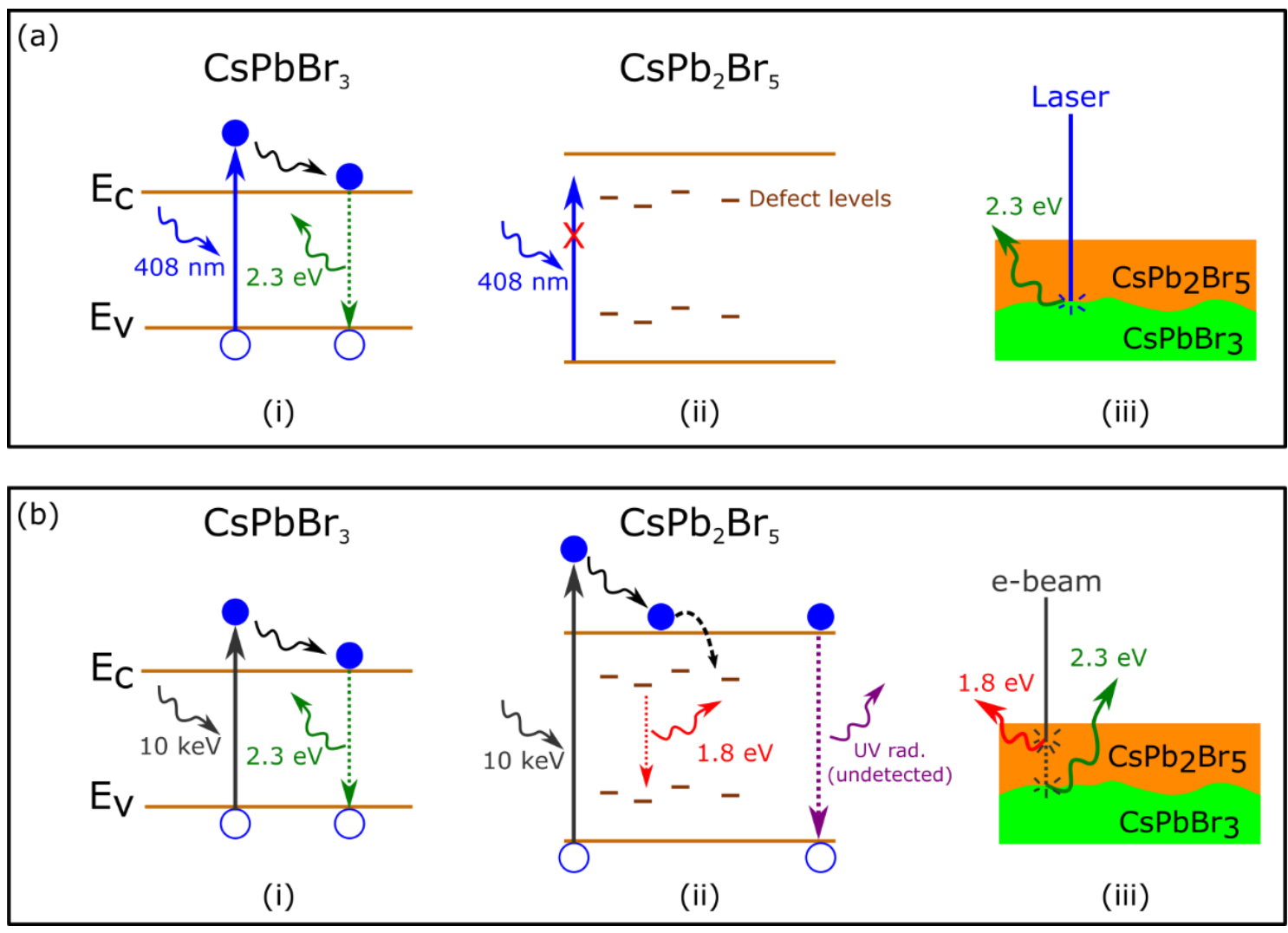

Figure S6. Schematic diagrams showing the differences between (a) PL and (b) CL emission from the $\mathrm{CsPbBr}_{3}$ part of the film (i), and from the $\mathrm{CsPb}_{2} \mathrm{Br}_{5}$ part of the film (ii). In PL, the laser light cannot excite the $\mathrm{CsPb}_{2} \mathrm{Br}_{5}$ surface layer of the film. Therefore, the PL signal originates from radiative recombination at the $\mathrm{CsPb}_{2} \mathrm{Br}_{5} / \mathrm{CsPbBr}_{3}$ interface, where the $\mathrm{CsPbBr} 3$ effectively absorbs the light, as schematically drawn in (a-iii). In CL, the high-energy electron beam is able to excite the $\mathrm{CsPb}_{2} \mathrm{Br}_{5}$ surface layer. Band-to-band recombination of the $\mathrm{CsPb}_{2} \mathrm{Br}_{5}$ is expected, but above our detection capabilities. Radiative recombination via deep defects originates a broad, low-energy band at $1.8 \mathrm{eV}$, which CL mapping revealed, is localized to the $\mathrm{CsPb}_{2} \mathrm{Br}_{5}$. Therefore, the $\mathrm{CL}$ signal of the surface originates from defect-assisted, and band-to-band radiative recombination at the surface $\mathrm{CsPb}_{2} \mathrm{Br}_{5}$ and at the $\mathrm{CsPb}_{2} \mathrm{Br}_{5} / \mathrm{CsPbBr}_{3}$ interface respectively, as schematically shown in (b-iii)

\section{Integral breadth analysis}

The line profile of a diffraction peak obtained in our XRD experiments is a convolution of an instrumental and physical (domain size and strain) profiles. ${ }^{8}$ Removing the instrumental part is essential for XRD analysis. Since the determination of the entire profile is impractical for our purpose, we used simplified methods that depend on the measurement of profile widths (full width at half maximum, FWHM; or integral breadth, $\beta$ ) to remove the instrumental contribution. It is 
then possible to obtain the instrumental-corrected ("pure") profile width by assuming an analytical form for each profile. In the present work, we used parabolic approximation correction, ${ }^{8,9}$ which assumes that the instrumental broadening is described by a Gaussian function, and the "pure" profile by a Cauchy function:

$\beta=\beta_{0}\left(1-\frac{\beta_{i}^{2}}{\beta_{0}^{2}}\right)$

where $\beta$ is the "pure" breadth, $\beta_{0}$ and $\beta_{i}$ are the measured and instrumental breadths of the same Bragg peak. We measured the NIST-SRM 660c LaB6 standard as a reference material to obtain the instrumental breadth. The peaks of the diffractogram were fitted using Pseudo-Voigt functions and the obtained FWHMs was used to fit the Caglioti formula: ${ }^{10}$

$F W H M=\left(U \tan ^{2} \theta+V \tan \theta+W\right)^{\frac{1}{2}}$

where $U, V$ and $W$ are fitting parameters. Using these parameters and equation (2) we calculated the instrumental widths $F W H M_{i}$ at the position of the peaks of the sample. Since the instrumental broadening is considered Gaussian, we calculated the instrumental integral breadth, assuming a Gaussian shape as:

$\beta_{i}=\frac{F W H M}{2} \sqrt{\frac{\pi}{\ln 2}}$

We also fitted the peaks in the diffractograms of the mixed-phase films using pseudo-Voigt functions, and the sample' measured integral breadth $\left(\beta_{0}\right)$ was obtained as the ratio of the area of the fitted profile and the maximum intensity of the peak. Then, using equations (1) and (3) we obtained the "pure" integral breadth for domain size analysis.

To separate domain size and the strain broadenings, we used and compared three methods. ${ }^{8,9}$ The first one assumes that the Strain is approximated by a Gaussian profile, and the domain size by a Cauchy profile. The equation describing the relation between strain and domain size is: 
$\frac{\beta^{2}(2 \theta)}{\tan ^{2} \theta_{0}}=\frac{K \lambda}{L}\left[\frac{\beta(2 \theta)}{\tan \theta_{0} \sin \theta_{0}}\right]+16 e^{2}$,

where $\beta(2 \theta)$ is the instrumental broadening-corrected integral breadth, $\theta_{0}$ is the peak maximum position, $L$ is the domain size, $e$ is the "maximum strain", $\lambda$ is the $\mathrm{X}$-ray wavelength, and $K$ is a constant that for practical purposes can be set to one.

The second method assumes both broadenings to be approximated by Gaussian profiles, and the equation describing the relation between strain and domain size is:

$\beta^{2}(2 \theta) \cos ^{2} \theta_{0}=\left(\frac{\lambda}{L}\right)^{2}+16 e^{2} \sin ^{2} \theta_{0}$

The third method is the Williamson-Hall (WH) method, ${ }^{11}$ in which both strain and size broadening are approximated as Cauchy profiles. The resulting equation is:

$\beta^{2}(2 \theta) \cos \theta_{0}=\frac{\lambda}{L}+4 e \sin \theta_{0}$.

Equations (4) to (6) can be approximated to linear plots, from which the domain size $L$ can be determined. Figure S7 shows the scatter plots for separation of domain size and strain using the three methods described above. We used the diffraction peaks listed in Table $\mathrm{S} 1$ for $\mathrm{CsPbBr}_{3}$ and $\mathrm{CsPb}_{2} \mathrm{Br}_{5}$. The data points exhibit substantial scatter and do not allow fitting a line for the GaussianGaussian and WH methods, especially for low temperatures. The points calculated with the Cauchy-Gaussian method are less scattered and allow fitting of the line profile (Figure S7a). We used the slope obtained from these fits to estimate $L$.

Table S1. List of diffraction peaks used in the integral breadth analysis of domain size

$\mathrm{CsPbBr}_{3}$

$\mathrm{CsPb}_{2} \mathrm{Br}_{5}$

\begin{tabular}{lll}
\hline Orthorhombic & Tetragonal & Cubic \\
\hline
\end{tabular}

$002,112,004,202,213,002,110,004$

$220,310,330,226,415 \quad 220,024,330$

100, 001, 002,

200 ,

$212,003,211$

100,200 ,

300,320 
For the evaluation shown in Figure $3 b$ in the main text, we plotted $\frac{\beta^{2}(2 \theta)}{\tan ^{2} \theta_{0}}$ vs. $\frac{\beta(2 \theta)}{\tan \theta_{0} \sin \theta_{0}}$ with the available Bragg reflections of $\mathrm{CsPbBr}_{3}$ and $\mathrm{CsPb}_{2} \mathrm{Br}_{5}$ at each temperature. The domain size as a function of temperature was evaluated from the slopes, $\frac{\lambda}{L}$.

For the purpose of comparing the domain size, we also calculated $L$ using the Scherrer's equation:

$L=\frac{0.9 \lambda}{\beta \cos \theta_{0}}$

The domain size calculated using equation 7 exhibit a similar trend with temperature to that calculated with the Cauchy-Gaussian method. The domain size values calculated for the $\mathrm{CsPb}_{2} \mathrm{Br}_{5}$ vary between 17 and $50 \mathrm{~nm}$, and between 22 and $200 \mathrm{~nm}$ for $\mathrm{CsPbBr}_{3}$. These values serve only as an estimation of the coherently diffracting domain size, but due to the strong preferential orientation I the film, they cannot be considered quantitatively. Furthermore, with this approximation we neglect the effect that strain causes in the broadening of the peaks. 


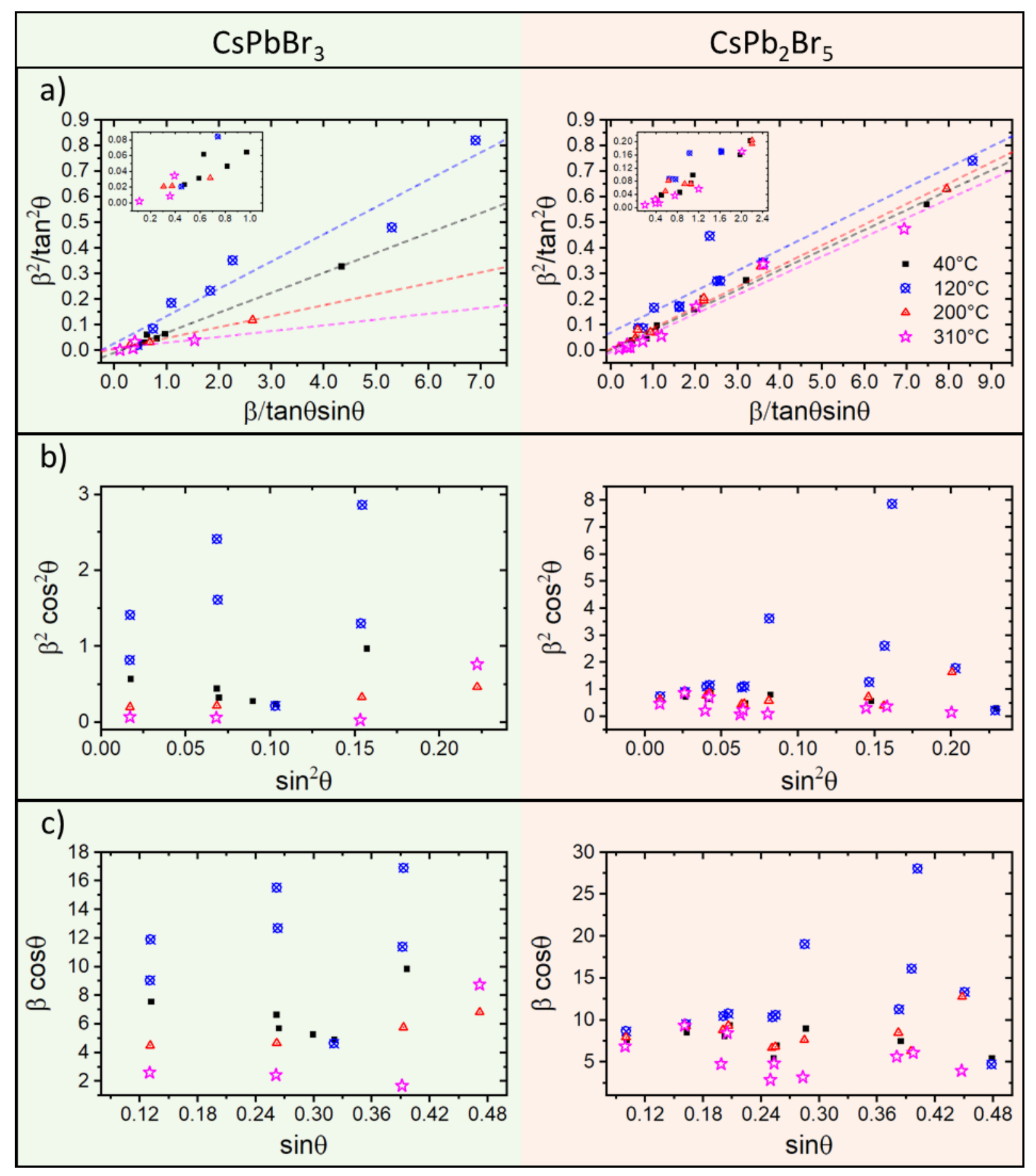

Figure S7. Domain size/strain separation calculation at different temperatures using (a) CauchyGaussian (equation 4), (b) Gaussian-Gaussian (equation 5), and (c) Williamson-Hall (CauchyCauchy, equation 4) approximations for the $\mathrm{CsPbBr}_{3}$ (green background) and $\mathrm{CsPb}_{2} \mathrm{Br}_{5}$ (orange background) phases in the mixed-phase film. For the Cauchy-Gaussian approximation, the calculated linear fits are also plotted. 


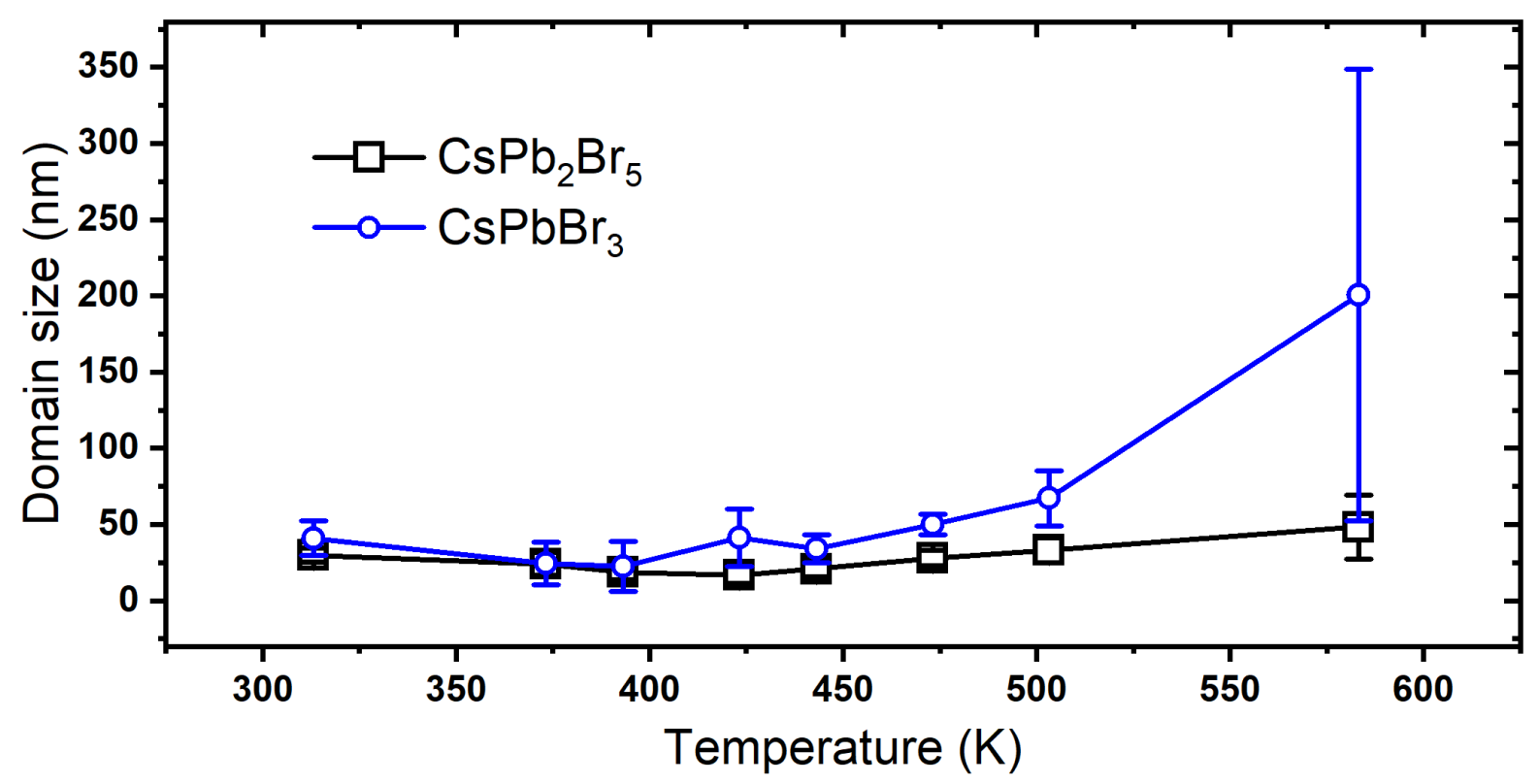

Figure S8. Domain size as a function of temperature, estimated using Scherrer's equation (7) for $\mathrm{CsPbBr}_{3}$ and $\mathrm{CsPb}_{2} \mathrm{Br}_{5}$ in the mixed-phase film.

\section{References}

(1) Caicedo-Dávila, S.; Funk, H.; Lovrinčić, R.; Müller, C.; Sendner, M.; Cojocaru-Mirédin, O.; Lehmann, F.; Gunder, R.; Franz, A.; Levcenco, S. et al. Spatial Phase Distributions in Solution-Based and Evaporated Cs-Pb-Br Thin Films. J. Phys. Chem. C 2019, 123 (29), 17666-17677.

(2) Rodová, M.; Brožek, J.; Nitsch, K. Phase Transitions in Ternary Caesium Lead Bromide. J. Therm. Anal. 2003, 71 (62), 667-673.

(3) Cola, M.; Riccardi, R. Binary Systems Formed by Lead Bromide with (Li, Na, K, Rb, Cs and TI) Br: A DTA and Diffractometric Study. Zeitschrift für Naturforsch. A 1971, 26, 1328.

(4) Moller, C. K. The Structure Of Perovskite-Like Cæsium Plumbo Trihalides. Mat. Fys. Medd. Dan. Vid. Selsk 1959, 32 (2). 
(5) Everhart, T. E.; Hoff, P. H. Determination of Kilovolt Electron Energy Dissipation vs Penetration Distance in Solid Materials. J. Appl. Phys. 1971, 42 (13), 5837-5846.

(6) $\mathrm{CsPb}_{2} \mathrm{Br}_{5}$ Crystal Structure: Datasheet from "PAULING FILE Multinaries Edition -- 2012"' in SpringerMaterials." Springer-Verlag Berlin Heidelberg \& Material Phases Data System (MPDS), Switzerland \& National Institute for Materials Science (NIMS), Japan.

(7) Yin, J.; Yang, H.; Song, K.; El-Zohry, A. M.; Han, Y.; Bakr, O. M.; Brédas, J. L.; Mohammed, O. F. Point Defects and Green Emission in Zero-Dimensional Perovskites. J. Phys. Chem. Lett. 2018, 9 (18), 5490-5495.

(8) Klug, H. P.; Leroy E. Alexander. X-Ray Diffraction Procedures for Polyerystalline and Amorphous Materials, Second.; Wiley, 1974.

(9) Varin, R. A.; Bystrzycki, J.; Calka, A. Effect of Annealing on the Microstructure, Ordering and Microhardness of Ball Milled Cubic $\left(\mathrm{L1}_{2}\right)$ Titanium Trialuminide Intermetallic Powder. Intermetallics 1999, 7 (7), 785-796.

(10) Caglioti, G.; Paoletti, A.; Ricci, F. P. Choice of Collimators for a Crystal Spectrometer for Neutron Diffraction. Nucl. Instruments 1958, 3 (4), 223-228.

(11) Williamson, G. K.; Hall, W. H. X-Ray Line Broadening from Filed Aluminium and Wolfram. Acta Metall. 1953, 1 (1), 22-31. 